\title{
Promoting Sustainability Awareness through Energy Engaged Virtual Communities of Construction Stakeholders
}

\author{
Yacine Rezgui, Ian E. Wilson, and Haijiang Li \\ School of Engineering, Cardiff University, Queen's Buildings, The Parade, \\ CARDIFF, CF24 3AA, Wales, UK \\ RezguiY@cf.ac.uk, WilsonI@Cf.ac.uk, LiH@cf.ac.uk
}

\begin{abstract}
Sustainability requires the engagement of every single constituent of the "building" product supply chain, from concept design to operation. The research explores ways of creating circles of impacts that bind building professionals, energy administrations, and citizens in a shared low carbon experience through a "one-stop-shop" Sustainable Construction Service Platform. The paper discusses awareness raising, stakeholders' engagement, technical requirements, and adoption and diffusion factors related to the platform. The research identifies the need to use advanced knowledge management functionality to leverage the value dimension of the proposed "one-stop-shop" service platform initiative.
\end{abstract}

Keywords: Sustainability, Construction, Awareness Raising, Communities of interest, Knowledge Management.

\section{Introduction}

Construction stakeholders involved in new or refurbishment projects are faced with: (a) complex legislation related to low carbon buildings, (b) a plethora of overlapping commercial tools supporting the process of delivering low carbon buildings, (c) numerous guidelines and documentation related to low carbon buildings, (d) an increasingly rigorous energy certification process, and (e) lack of clarity on types of financial assistance and eligibility criteria [1]. Furthermore, while a great deal of expertise already exists in detailing and constructing low-energy buildings, much of this expertise is fragmented and exists in various forms, with no real systematic means or mechanisms to assist construction stakeholders in their low carbon decisionmaking [2]. In this context, it is imperative to make building energy expertise widely available; in particular during the early design stages of a project, when most of the key decisions that impact on energy are made.

Existing knowledge on sustainability is multi-disciplinary (i.e. concerns various specialties, including Architecture, Heating, Ventilation, Air conditioning, Electricity, Plumbing). It involves architectural and engineering sciences applied to the lifecycle of a building project from concept design to demolition. Sustainability knowledge is available in the form of, and embedded in, text documents, spreadsheets, drawings, 
images, video, and in some cases, relational databases (e.g. product libraries). It has a strong "tacit" dimension. It can be nurtured (shared, enhanced, and transferred) through discussion forums, story telling, or simple knowledge enrichment techniques such as annotation. In fact, sustainability goals can only be achieved if shared and value-added relevant resources of knowledge and expertise inform construction activities.

The paper describes research conducted in the context of the SCrIPt project that aims to assist and improve the capacity of building professionals to offer effective energy and low carbon solutions and increase the demand for such solutions, while at the same time adopting energy reducing use patterns in buildings. This has the potential to pave the way to better educated, more engaged and energy responsible/responsive building professionals and citizens. The paper summarizes the methodology that underpins the research, followed by a review of related work. The 'Sustainable Building Portal' prototype demonstrator is then briefly discussed, followed by key recommendations that emerged from consultation. Finally, the paper concludes with directions for future work.

\section{Methodology}

The research is exploratory in nature; the overall aim is to answer the following research question: Would construction stakeholders, organized into virtual communities, adopt and promote sustainability practices in projects by nurturing (sharing, creating, enriching, and transferring) sustainability knowledge through a Sustainable Construction Service Platform?

The project first developed a prototype demonstrator that was then used as a guide to gather consensus on the requirements for such a "one-stop-shop" platform.

The industry consultation involved three workshops; the first two (Initial Scoping Workshop and Consensus Building Workshop) were hosted by the project partners. The third was hosted by the Low/Zero Carbon Hub in Wales (Wales LZC Hub) and was attended by the project partners. The Wales LZC Hub is a coalition that is supported by the Welsh Assembly Government (WAG), the building industry, housing, and voluntary sectors, to help meet WAG's target of making new buildings zero carbon from 2011 and to help Wales meet its goal of reducing carbon dioxide emissions by three per cent a year from 2011.

The findings from these workshops fed forward to a series of interviews and also to the recommendations for the proposed "one-stop-shop" Sustainable Construction Service Platform. The combined results from these methodological elements allowed the formulation of key recommendations for the full development of the platform.

\section{Related Work}

The construction sector is complex and is characterized by a culture and organizational environment that differentiate it from many other industrial sectors [3,4,5]. As reported in [6,7], many KM initiatives in construction have failed because they: (a) relied on employees' willingness to share knowledge, (b) completely 
overlooked the social dimension of KM, and (c) could not make sense of much of the data and information produced and managed by legacy software applications. The low take up and use of advanced ICT solutions for KM and information sharing in general are due to various issues including:

$>$ Problems of technology adoption can have a negative effect on individual satisfaction with the team experience and performance. Conversely, when team members are able to deal with technology related challenges, high trust develops, which promotes a culture of knowledge sharing.

$>$ Rather than widely sharing valuable knowledge, much of which is tacit and nurtured in small social networks, cultural restraints engender "silos" of isolated knowledge.

$>$ Lack of awareness about the value in sharing knowledge and experiences.

$>$ Off-the-shelf commercial solutions often fail to deliver as they tend neither to accommodate existing practices, nor to build on existing corporate solutions.

$>\mathrm{KM}$ solutions tend to lack flexibility and scalability as the needs of the organization and users evolve.

$>$ A lack of shared language holding a common understanding of concepts across disciplines prevents effective communication and experience sharing.

$>$ Access to knowledge from construction sites is essential, but restricted by technical limitations.

$>$ Employees and organizations feel reluctant to share knowledge when security, confidentiality, and IPR concerns are not properly addressed.

These issues engender reluctance and a resistance to adopt technology [5], and hence access useful information and knowledge available electronically. Difficulty of use and lack of perceived usefulness of ICT solutions are also due to limited search facilities, information overload, etc., and, crucially, lack of structure in existing documents.

The authors analysed a number of relevant web sites to look at the nature of their content, including "sustainability", and ways to access the information. The chosen sites cover the construction sector, sustainable building, and/or low carbon issues [6].

Overall, whilst there is a plethora of information available regarding sustainable construction, there is no central location that appears to be authoritative and comprehensive. Also, the various portals operate on an "information push" basis and there is little or no attempt to capture the user experience. Moreover, on viewing these various sites in the process of searching for sustainable construction information there is a feeling of confusion and uncertainty in what constitutes the best and particularly the most important information.

\section{The Sustainable Building Portal Demonstrator}

The Sustainable Building Portal demonstrator, which takes a 'top-down' approach to information provision, aims at facilitating and structuring access to existing (codified and available in electronic form) sustainability knowledge. The portal aims to be 
"Wales' official guide to help the construction industry minimize the impact of buildings on the environment" and to become the primary source of reference for sustainable construction. It comprises ten main sections covering: Sustainable Design; Costs \& Financial Support; Legislation \& Policy; Guides, Codes \& Articles; Wales LZC Hub; Project Team; Training \& Skills; Buildings \& Infrastructure; Materials \& Products; Research \& Development.

A solution such as the Sustainable Building Portal, provided that it is widely used (becoming a pervasive process), constitutes a starting point for centralized information sharing, but much of the information required by many practitioners is either unavailable to them because they do not have the correct technical services which may be due to cost limitations, inadequate ICT literacy, lack of training, etc. or perhaps is buried in documents that, due to a lack of contextualization, do not appear to be relevant to them.

\section{Results from the Consultation}

The consultation confirmed the fact that sustainability knowledge in construction is fragmented, diverse, embedded in various documents, and developed in a nonconcerted and integrated way across stakeholders, localities, regions, and countries. It also highlighted a number of issues related to the Sustainable Building Portal demonstrator, including:

$>$ Lack of sharing, exploitation, and reuse of isolated sustainable practices and principles acquired through practice across the industry.

$>$ Lack of education and awareness across key construction stakeholders and building end-users.

$>$ Lack of easy access to structured sustainability information and knowledge.

$>$ Unclear links between sustainability principles and current construction regulations and standards. In fact, there is no explicit statutory requirement that the Building Regulations should cover sustainability, even though many of the approved documents already do so. This is a substantial statutory barrier to progress on the sustainability of buildings.

$>$ Uncertainty about the cost of sustainable solutions / technologies.

The notion of creating a "Map of Everything" emerged from the Wales LZC Hub workshop and was the most highly weighted issue of immediate concern to the delegates. The Sustainable Building Portal is perceived as a starting point for centralized information sharing, but what is required is a more complete KM solution, which incorporates a 'bottom-up' approach to sustainable construction information and thereby its transformation to knowledge.

The major themes that emerge from the fieldwork may be summarized according to three categories that inform recommendations for a fully workable solution: Information Requirements; Functionality/Services; Development and Management. These recommendations to ensure a feasible solution, and which will need to be addressed in a follow-up to this study, are as follows: 


\subsection{Information Requirements}

$>$ Categorize information: various different information types and topics need to be catered for, including regulations, legislation, research and innovation, etc.;

$>$ Cultivate and promote a shared perception of sustainable construction: there is need for a consistent 'message' to mitigate uncertainty and to ensure that all stakeholders (including end users and consumers) are in concord;

$>$ Provide a reliable and verified repository of 'basics' information: possibly the 'top 10 most important documents' to alleviate current misconceptions;

$>$ Dedicated and focused best practice information: currently much of this information is very 'high level'; consider inclusion of (anonymous) failures;

$>$ Information and knowledge management and sharing: there is a need to transform 'information' into knowledge, which requires advanced content management, and contextualization of new concepts;

$>$ Real data provision: there is currently a lack of empirical data for Wales, including costs data, which need to be collected/disseminated across the sector;

$>$ Provide avenues of marketing, or connectors: data and information regarding supply chain, products, etc. are required;

$>$ "Map of Everything": include this result from the Wales LZC Hub once it is completed, or a clearly visible link to it.

\subsection{Functionality/Services}

$>$ Smart Searching Facilities: current searching methods are unsatisfactory and limited, treating documents as 'black boxes' and lacking filtering mechanisms.

$>$ Create and implement a Sustainable Construction Ontology: this will act as the kernel of the portal and will support smarter functionality, including search engine;

$>$ User Profiling: this service will enrich users' experiences, and will increase user adoption and participation;

$>$ News Service(s): including filtering, searching capabilities, and optional automatic news 'push' (notification) for key developments that are of direct interest to a particular user, with timely delivery. This will rely on both the Ontology and User Profiling;

$>$ Bi-directional channels for information sharing and enrichment: incorporating wiki concepts and e-Forums. This will rely on both the Ontology and User Profiling;

$>$ Interface for shared tools/services: free to use tools and potential for paid tools possibly on a rental basis. Also potentially include a tools register and/or tools market watch;

$>$ CPD facilities: to potentially include educational tools such as live and/or interactive case studies, and with clear information on access to skills training programmes, educational courses, etc.

$>$ Value added tools and services: "one-stop-shop" access to energy tools and related services, including Energy compliance checking tools. 


\subsection{Development and Management}

$>$ Focus and scope: the area under investigation is vast, and therefore requires a clear agreement of the ultimate aims and objectives, starting from an initial focus for concentration of development covering a manageable scope;

$>$ Iterative and Incremental Development: a clear 'project plan' will be required to ensure that development proceeds according to a realistic timeframe. Initially doing one thing really well and subjecting it to testing and validation will ensure 'proof of concept' before proceeding to expand the platform. Requirements and functionality will almost inevitably change, but it is important to stick to the remit;

$>$ Continual consultation: end-users should be involved in the development process from the beginning; industry consultation will provide real-world feedback;

$>$ Partners: a broad spectrum of partners (whether 'full' partners or consultation partners) should be agreed as soon as possible. The full proposed platform will need expertise from various disciplines;

$>$ Define ownership: this includes who pays for maintenance of the developed solution, and also addressing IPR issues;

$>$ Management team: to manage information quality; moderation (e.g. on forums); continual management of information content to ensure accuracy, trustworthiness, and consistency;

$>$ Management plan: to manage resources and financing; costs have to be addressed for maintenance of the platform after development and implementation - it may cost at least as much per year to run as to build, and it is essential that it is kept up to the minute to maintain credibility;

$>$ Marketing and Dissemination: plans need to be implemented to ensure wide awareness raising and acceptance and use of the developed solution;

$>$ Involve intermediaries: e.g. builders merchants and other communities of interest for those who do not frequently use/have access to the internet.

\section{Conclusion}

Several construction-related information portals have been commissioned and developed by various institutions at a UK (including Wales) and European level. The information published by these portals is in many cases redundant, overlapping, specialized, and not easy to locate and access [6]. Furthermore, these portals do not provide user friendly and effective knowledge management functionality, including smart search facilities, which results in low adoption from construction stakeholders.

Moreover, the research highlighted the fact that existing sustainability knowledge suffers from a number of problems, and that sustainability goals can only be achieved if shared and value-added relevant resources of knowledge and expertise inform construction activities. The research has clearly established the need for a number of requirements and user-centred services that can enhance the current Sustainable Building Portal. The Authors believe that the development of these services, in line with the industry consultation results, clearly defines the need for a fully workable 
version of the Sustainable Building Portal that can be developed using state-of-the-art Web 2.0 technologies, using a web service approach [9] underpinned by a low carbon ontology. A consortium has been put together, involving the Wales LZC Hub, BRE Wales, and Constructing Excellence in Wales, and a number of industrial organizations that took part in the consultation, to deliver the above upgraded version of the Sustainable Building Portal. This will be reported in follow-on publications.

\section{References}

[1] Rezgui, Y., Miles, J.C.: Exploring the potential of SME alliances in the construction sector, Construction Engineering and Management. Journal of the American Society of Civil Engineering (2010), 10.1061/(ASCE)CO.1943-7862.0000150

[2] Rezgui, Y.: Knowledge systems and value creation: An action research investigation. Industrial Management \& Data Systems 107(2), 166-182 (2007)

[3] Wetherill, M., Rezgui, Y., Boddy, S., Cooper, G.S.: Intra- and interorganizational knowledge services to promote informed sustainability practices. Journal of Computing in Civil Engineering 21(2), 78-89 (2007)

[4] Boddy, S., Rezgui, Y., Cooper, G., Wetherill, M.: Computer integrated construction: A review and proposals for future directions. Advances in Engineering Software 38(10) (2007)

[5] Rezgui, Y.: Exploring Virtual Team-Working Effectiveness in the Construction Sector. Interacting with Computers 19(1), 96-112 (2007)

[6] Rezgui, Y., Wilson, I., Miles, J.C., Hopfe, C.J.: Federating information portals through an ontology-centered approach: A feasibility study. Advanced Engineering Informatics (2010), 10.1016/j.aei.2010.02.001

[7] Rezgui, Y., Hopfe, C.J., Vorakulpipat, C.: Generations of knowledge management in the architecture, engineering and construction industry: An evolutionary perspective. Advanced Engineering Informatics (Elsevier) (2010) 10.1016/j.aei.2009.12.001

[8] Vorakulpipat, C., Rezgui, Y., Hopfe, C.J.: Value Creating Construction Virtual Teams:A Case Study in the Construction Sector, Automation in Construction (Elsevier) (2009), 10.1016/j.autcon.2009.11.016

[9] Rezgui, Y.: Role-based service-oriented implementation of a virtual enterprise: A case study in the construction sector. Computers in Industry 58(1), 74-86 (2007) 